\title{
Benefits of Case-Based versus Traditional Lecture-Based Instruction in a Preclinical Removable Prosthodontics Course
}

\author{
David B. Samuelson, DDS; Kimon Divaris, DDS, PhD; Ingeborg J. De Kok, DDS, MS \\ Abstract: This study compared the acceptability and relative effectiveness of case-based learning (CBL) versus traditional \\ lecture-based (LB) instruction in a preclinical removable prosthodontics course in the University of North Carolina at Chapel Hill \\ School of Dentistry DDS curriculum. The entire second-year class ( $\mathrm{N}=82)$ comprised this crossover study's sample. Assessments \\ of baseline comprehension and confidence in removable partial denture (RPD) treatment planning were conducted at the begin- \\ ning of the course. Near the end of the course, half of the class received CBL and LB instruction in an RPD module in alternating \\ sequence, with students serving as their own control group. Assessments of perceived RPD treatment planning efficacy, compre- \\ hension, and instruction method preference were administered directly after students completed the RPD module and six months \\ later. Analyses of variance accounting for period, carryover, and sequence effects were used to determine the relative effects of \\ each approach using a $p<0.05$ statistical significance threshold. The results showed that the students preferred CBL ( $81 \%$ ) over \\ LB instruction (9\%), a pattern that remained unchanged after a six-month period. Despite notable period and carryover effects, \\ CBL was also associated with higher gains in RPD treatment planning comprehension $(\mathrm{p}=0.04)$ and perceived efficacy $(\mathrm{p}=0.01)$ \\ compared to LB instruction. These gains diminished six months after the course - a finding based on a $49 \%$ follow-up response \\ rate. Overall, the students overwhelmingly preferred CBL to LB instruction, and the findings suggest small albeit measurable \\ educational benefits associated with CBL. This study's findings support the introduction and further testing of CBL in the pre- \\ clinical dental curriculum, in anticipation of possible future benefits evident during clinical training.
}

Dr. Samuelson is an Advanced Education in General Dentistry Resident, Veterans Affairs Medical Center, Fayetteville, NC; Dr. Divaris is Associate Professor, Department of Pediatric Dentistry, School of Dentistry and Department of Epidemiology, Gillings School of Global Public Health, University of North Carolina at Chapel Hill; and Dr. De Kok is Associate Professor, Department of Prosthodontics, School of Dentistry, University of North Carolina at Chapel Hill. Direct correspondence to Dr. Ingeborg J. De Kok, School of Dentistry, University of North Carolina, 336 Brauer Hall, CB\#7450, Chapel Hill, NC 27599-7450; 919-537-3963; ingeborg_dekok@unc.edu.

Keywords: dental education, teaching methods, assessment, prosthodontics

Submitted for publication 5/25/16; accepted 9/20/16

doi: $10.21815 / J D E .016 .005$

B oth dental faculty members and students have pointed to deficiencies and areas for improvement in dental curricula. ${ }^{1-3}$ One such deficiency is the lack of self-directed or active learning in most dental curricula, so that incorporation of enhanced, active learning methodologies has gained support. In active learning, students are placed at the center of the educational process, with a focus on the process of learning. ${ }^{3}$ In this methodology, students are engaged in activities, information-gathering, and higher order thinking including analysis, synthesis, and evaluation. These activities have been favored by dental students in diverse settings, are considered important elements of a positive academic environment, and have been found to be associated with improved learning outcomes. ${ }^{3-6} \mathrm{In}$ a recent review of problem-based (PBL) and case-based learning (CBL) in the medical and dental educational literature, Nadershahi et al. argued for the use of CBL due to its favorability with students and instructors, as well as its demonstrated "positive relationship with the development of clinical skill." 7

Removable partial denture (RPD) treatment planning is a potentially difficult part of dental curricula because students are required to understand and then translate a considerable volume of new information into clinical proficiency. At the same time, RPD treatment involves a great deal of concept integration and critical thinking. Prosthodontic faculty members at the University of North Carolina at Chapel Hill School of Dentistry had noted that students struggled in both preclinical courses and the provision of clinical care to properly design and sequence treatment plans for patients receiving treatment with RPD appliances. This has been attributed to students' inability to engage in multidisciplinary concept integration and translation into clinical application. To address this issue, an educational 
intervention to enhance the previously exclusively lecture-based instruction was planned.

Due to its demonstrated benefits, ${ }^{7-13} \mathrm{CBL}$ was chosen as the educational intervention for the removable prosthodontics preclinical course, and this study was designed to accompany its implementation. The aim of the study was to compare CBL with a traditional lecture-based (LB) approach as instructional modalities to facilitate student learning during the RPD treatment planning module. A similar study recently investigated the effectiveness of teambased learning versus LB in an RPD course at the University of Florida, but that study compared the results of different (consecutive) classes (2013 and 2014) of dental students. ${ }^{12}$ In our study, we sought to examine CBL's educational efficacy employing a crossover study design in a single DDS class and to specifically measure students' performance on RPD treatment planning comprehension, as well as students' perceived efficacy (confidence). Students' acceptance and preference of these instructional methods were also measured.

\section{Methods}

The study was reviewed by the University of North Carolina at Chapel Hill Office of Human Research Ethics (\#14-0720) and was determined to be exempt from further review. The DDS2 class $(\mathrm{N}=82)$ of the 2013-14 academic year served as the study population. The removable prosthodontics course for DDS2 students took place during the summer semester of 2014 and was of nine weeks' duration. A schematic outlining the study's crossover design is shown in Figure 1. A crossover design was chosen to allow all students to receive the intervention (CBL) while serving as their own control group (experiencing the traditional lecture).

\section{Timeline of Assessments and Module}

In week $1\left(\mathrm{~T}_{0}\right)$ of the course, all DDS2 students were given the following baseline assessments: treatment planning comprehension $\left(\mathrm{TPCME}_{0}\right)$, treatment planning confidence $\left(\mathrm{TPCFE}_{0}\right.$ ), and perceived knowledge assessment $\left(\mathrm{PKNE}_{0}\right)$. In weeks 2-7, all students participated in the course, which employed both traditional, didactic lectures and preclinical laboratory exercises. During this time, all students received identical instruction in each learning module.
In week $8\left(\mathrm{~T}_{1}\right)$, students began the RPD treatment planning module - the final module of the course. The class was split alphabetically into two cohorts: group $\mathrm{A}(\mathrm{n}=41)$ and group $\mathrm{B}(\mathrm{n}=41)$. Group $\mathrm{A}$ was further split into four small groups ( $n \sim 10)$, each of which participated in CBL activities. At the same time, group B received LB instruction. After those sessions, group A and group B took three identical assessments: treatment planning comprehension $\left(\mathrm{TPCME}_{1}\right)$, treatment planning confidence $\left(\mathrm{TPCFE}_{1}\right)$, and method acceptance assessment ( $\left.\mathrm{MAE}_{1}\right)$.

In week $9\left(T_{2}\right)$, the groups were switched, with group A receiving LB instruction and group B further split into small groups $(\mathrm{n} \sim 10)$ before participating in CBL activities. After this intervention, both group $\mathrm{A}$ and group $\mathrm{B}$ were given four assessments: treatment planning comprehension $\left(\mathrm{TPCME}_{2}\right)$, treatment planning confidence ( $\mathrm{TPCFE}_{2}$ ), method acceptance assessment $\left(\mathrm{MAE}_{2}\right)$, and method preference assessment $\left(\mathrm{MPE}_{2}\right)$.

To examine possible lasting effects of the intervention, we examined the students again six months after the end of the course. In the spring semester of $2015\left(\mathrm{~T}_{3}\right)$, all students in the original cohort $(\mathrm{n}=82)$ were invited to retake three assessments as a means of providing extended follow-up data: treatment planning comprehension ( $\mathrm{TPCME}_{3}$ ), treatment planning confidence ( $\mathrm{TPCFE}_{3}$ ), and method preference assessment $\left(\mathrm{MPE}_{3}\right)$.

\section{Instructional Methodologies and Details of Assessment}

The LB instruction consisted of a traditional, lecture-based presentation that covered the required treatment planning material according to the course objectives, delivered by the course director (third author); the same lecture was given to both groups. The CBL intervention was organized into four small groups ( $\sim 10$ students each), led by a teaching team comprised of an experienced prosthodontics faculty member and a third-year prosthodontics resident. The instructional methodologies consisted of one two-hour session for each modality (CBL or LB) and were exclusively clinical case discussion-driven. The prosthodontics faculty members and residents who participated as group leaders were given the same cases to discuss and scripts to follow prior to the CBL small-group sessions. The same cases were presented in the second session. The script contained description of the case, including medical history and its relevance related to treatment, consultations needed, 


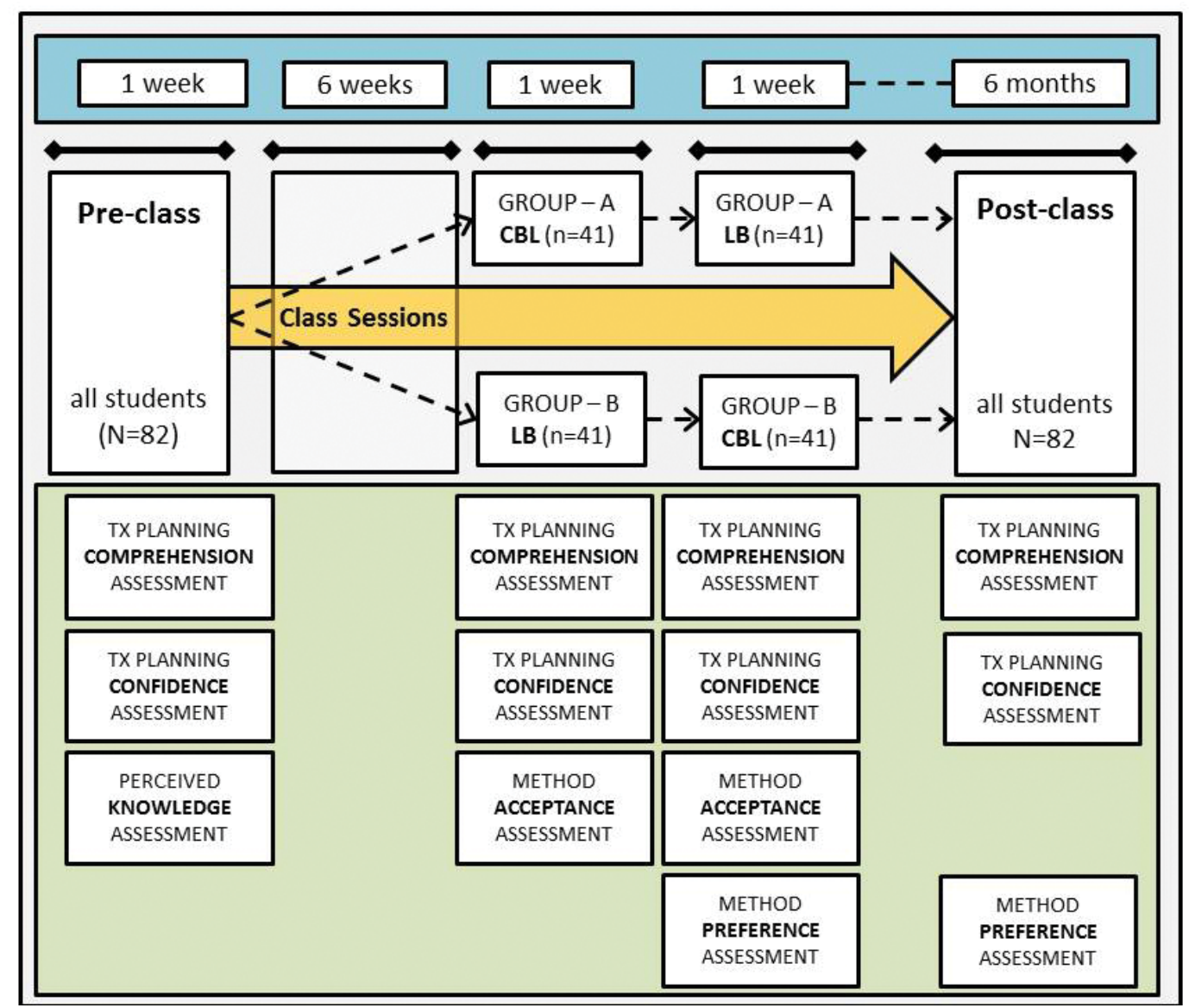

Figure 1. The 2x2 crossover study design evaluating acceptability and efficacy of case-based (CBL) learning versus lecture-based (LB) instruction during nine-week course and six months after

treatment plan with alternatives/potentially different scenarios, potential number of visits, and sequence of treatment (including biopsies, extractions, root canals, crowns, and removable prostheses). This information was provided to form an initial knowledge base and was accompanied with questions to further guide the discussion of each case.

Students' RPD treatment planning comprehension was this study's primary outcome; it was objectively assessed with RPD treatment planning comprehension assessments at four time points. These assessments were developed by the content expert and course director. In each assessment, students were presented with a clinical case and were then asked to identify and sequence appropriate treatment steps for comprehensive care including RPD. Assessments were given via an online learning management system (Sakai), and students were instructed to work alone on each assessment. All students were given the same clinical case and assessment at each of the four time points $\left(\mathrm{T}_{0}-\mathrm{T}_{3}\right)$. However, a different case was used at each time point, with one exception: the case given at $T_{0}$ was identical to the case given at $T_{3}$ so that a pre- and post-course evaluation could be conducted.

Assessment of performance on these assessments was done on a $0-100$ scale, where 0 indicated the lowest possible and 100 the highest possible RPD treatment planning comprehension. Comprehension was measured by development of a treatment plan that included the patient's restorative dental needs, Kennedy classification, RPD design including teeth to serve as abutments and major connector chosen, and sequencing visits to execute the plan. All cases at all time points were scored by the same graders. Assessments were obtained at the beginning of the course $\left(\mathrm{TPCME}_{0}\right)$ to assess baseline knowledge; after each instructional modality (TPCME $\mathrm{T}_{1}$ and $\mathrm{TPCME}_{2}$ ) 
to assess gains in comprehension after $\mathrm{CBL}$ and $\mathrm{LB}$ activities; and after the end of the course, during spring semester 2015 ( $\mathrm{TPCME}_{3}$ ), to assess retention.

The students' perceived RPD treatment planning efficacy/confidence was one of the study's secondary outcomes and was assessed as reported by the students at the same four time points: baseline (TPCFE $\mathrm{T}_{0}$ ), after delivery of each instructional modality (TPCFE ${ }_{1}$ and $\mathrm{TPCFE}_{2}$ ), and after the end of the course $\left(\mathrm{TPCFE}_{3}\right.$ ). A visual-analogue scale (VAS) was used to assess this domain, where a score of 0 indicated the least and 10 the most efficacy/confidence. The item to which students were asked to respond was as follows: "How confident are you in your ability to appropriately treatment plan a patient for removable partial prosthodontic treatment?"

Perceived contextual preparedness was assessed at the beginning of the course (at $\mathrm{T}_{0}$ only, $\mathrm{TPKNE}_{0}$ ) as a measure of students' perceived baseline knowledge derived from prosthodontics courses during the first years of study. A VAS was used to assess this domain, where a score of 0 indicated the lowest and 10 the highest perceived contextual preparedness/knowledge. The item used to assess this domain was as follows: "Based on your experiences in previous prosthodontics courses, how well prepared do you feel to take Removable Partial Prosthodontics?"

Instructional method acceptance was assessed directly following each LB and CBL activity to determine students' perceived benefit and acceptability of the activities $\left(\mathrm{MAE}_{1}\right.$ and $\left.\mathrm{MAE}_{2}\right)$. This domain was assessed with the same VAS 0-10 scale on the following question: "How beneficial was today's session with regard to your development of skills required for RPD treatment planning?" Instructional method preference was assessed twice: after students attended both the LB and CBL modules during the last week of the course (MPE ${ }_{2}$ ) and after the end of the course in spring $2015\left(\mathrm{MPE}_{3}\right)$. A multiple-choice question ("Which of the following instructional methods did you most prefer?") was used to assess this domain, with the available options of no preference, small group case discussion, and traditional lecture.

\section{Analytical Strategy}

Our analytical approach was based upon recommended methods for analysis of crossover study designs. ${ }^{14}$ Descriptive statistics were used to determine the proportion of students reporting their method preference and acceptance. Analyses of vari- ance were implemented with Stata's (StataCorp LP, College Station, TX, USA) pkcross routine for the continuous study outcomes (RPD treatment planning comprehension and perceived efficacy), accounting for the $2 \times 2$ crossover study design features. These included intersubject effects (due to the alternating sequence of instructional method administration between groups A and B) and intrasubject effects attributable to the 1) differing instructional "method effect"; 2) instructional method effect that carries over from $\mathrm{T}_{1}$ to $\mathrm{T}_{2}$; and 3 ) "study period" design feature, wherein students in groups A and B may differ systematically due to their familiarity with the study (or course and assessment) conditions.

In terms of statistical power, the study was carried out among a fixed population of 82 students (entire DDS2 class, split into two groups), so determination of the sample size was not driven by a power calculation. However, using the proposed experimental design and assuming a mean comprehension score of 80 (standard deviation $=10$, we used $\mathrm{G}$ *Power to estimate over $80 \%$ power of detecting differences of $\geq 3.5$ points magnitude at the 0.05 alpha level. ${ }^{15}$ Visualizations of score distributions over time were done using descriptive plots ("strip plots") using Stata 14.1 , and a conventional $\mathrm{p}<0.05$ threshold was used for statistical significance.

\section{Results}

Students overwhelmingly preferred the CBL small-group seminars $(81 \%)$ over LB instruction $(9 \%)$, and few had no preference (10\%). This pattern remained unchanged after a six-month period (Figure 2). Notable increases were noted in both subjective (perceived treatment planning efficacy, Figure 3) and objective (treatment planning comprehension, Figure 4) measures due to RPD instruction. Both methods were associated with substantial gains in comprehension (CBL: 31.7 points, LB: 26.2 points).

Despite notable period and carryover effects (Table 1), CBL was associated with higher gains in treatment planning comprehension ( $\mathrm{p}=0.04$; an absolute difference of 5.5 points on a 100-point scale or a $21 \%$ relative increase compared to LB). Similarly, $\mathrm{CBL}$ was associated with greater gains in perceived efficacy ( $p=0.01$; absolute difference of 0.7 points on a ten-point scale or $19 \%$ relative increase compared to LB instruction). These gains diminished six months after the course; however, this estimate was based on a $49 \%$ follow-up response rate. 


\section{Discussion}

In this study among a class of 82 second-year dental students, we found evidence of objectively measured and subjectively reported educational benefits associated with the implementation of CBL instruction relative to a traditional LB approach in the context of a removable prosthodontics course. Although the actual quantitative gains in assessments of RPD treatment planning comprehension and confidence were modest (about 20\% for both) and diminished approximately six months after the end of the course, students overwhelmingly preferred the case-based methodology. Taken together, these results provide support for the implementation of CBL modules in dental education, but future studies should clarify what additional modalities and educational experiences must be in place to maximize the retention of learned skills and their transfer to clinical stages of dental education.

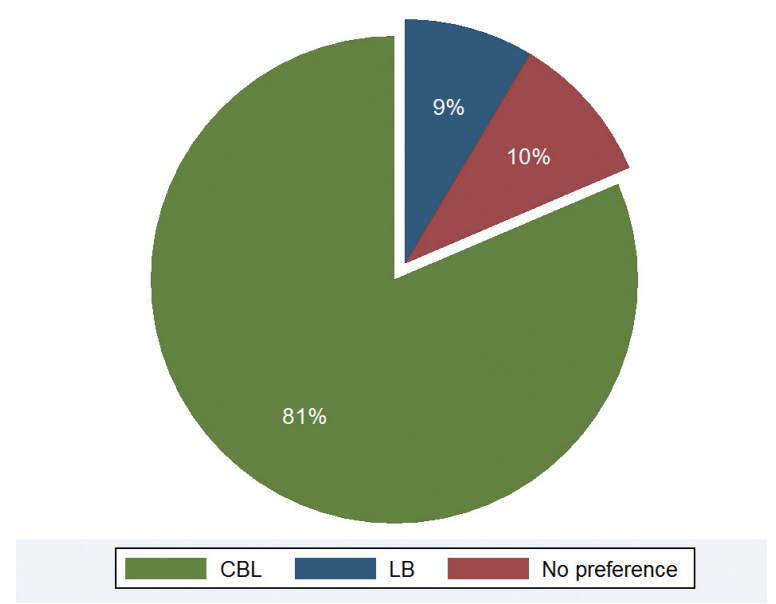

Figure 2. Students' $(\mathrm{N}=81)$ preference for case-based learning $(\mathrm{CBL})$ versus lecture-based $(\mathrm{LB})$ instruction in removable partial denture module after exposure to both approaches

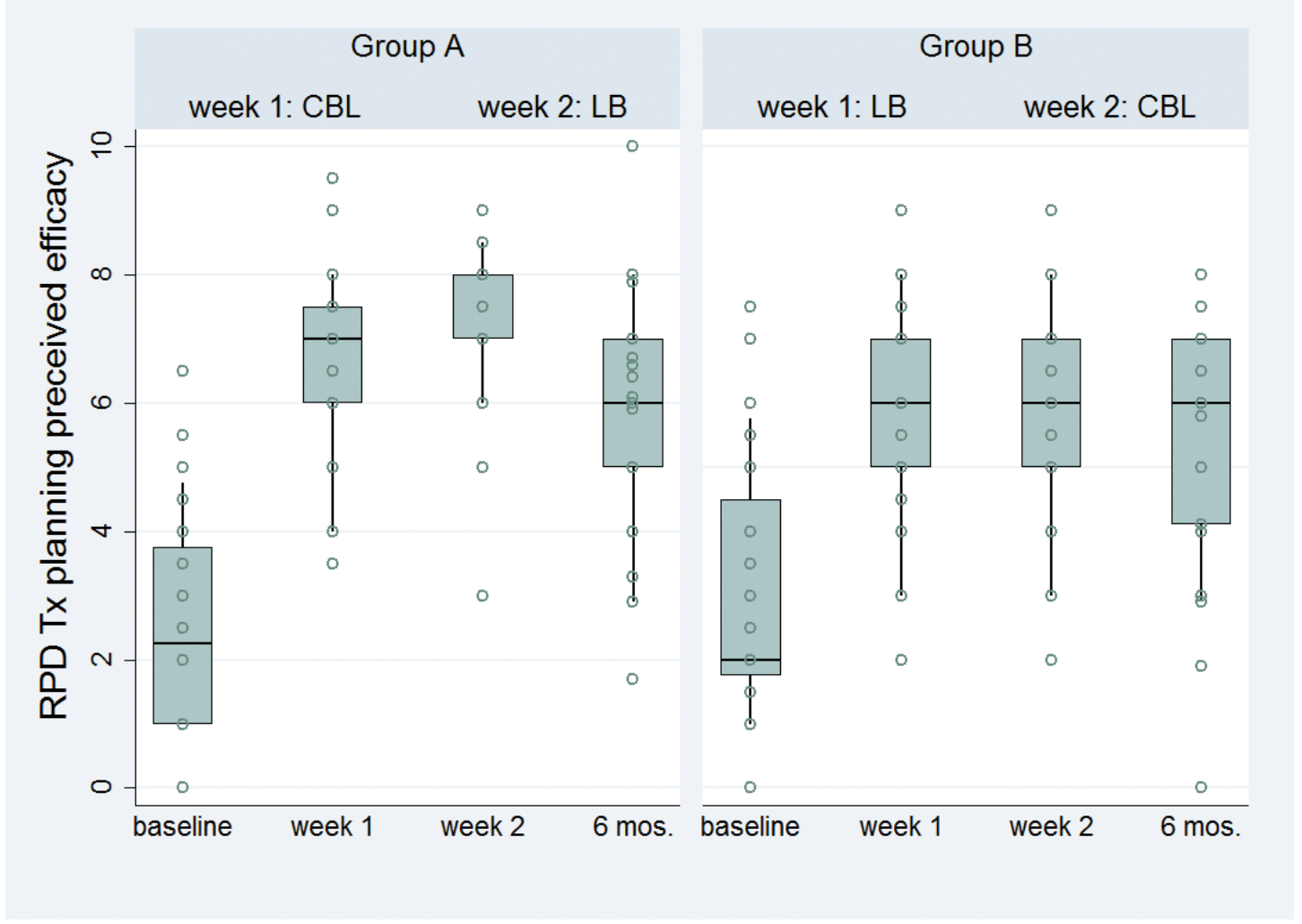

Figure 3. Students' self-reported efficacy in removable partial denture (RPD) treatment planning during course and six months after, according to instruction method sequence

$\mathrm{CBL}=$ case-based learning; $\mathrm{LB}=$ lecture-based instruction 


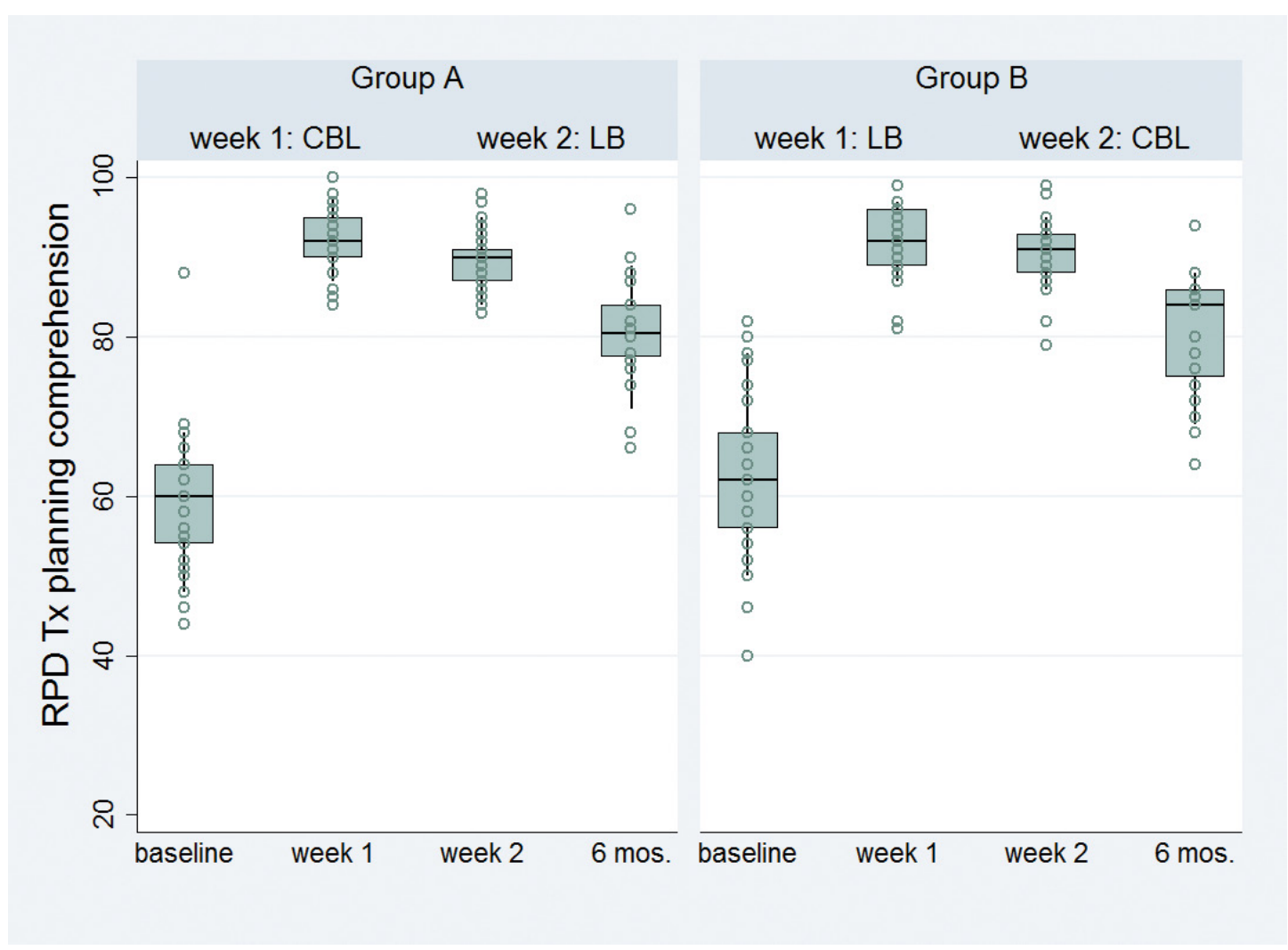

Figure 4. Students' objectively assessed removable partial denture (RPD) treatment planning comprehension during course and six months after, according to instruction method sequence

$\mathrm{CBL}=$ case-based learning; $\mathrm{LB}=$ lecture-based instruction

It has been suggested that CBL can stimulate independent learning and may facilitate competency development in a manner that parallels students' future professional lives. ${ }^{8}$ Our findings were consistent with recent reports demonstrating the multilevel benefits of CBL in predoctoral dental education. ${ }^{9-12}$ In addition, evidence from medicine suggests that CBL may be favored over problem-based learning (PBL), as the former represents a form of "guided inquiry" versus the less structured "open inquiry" philosophy inherent in PBL. ${ }^{13}$ A 2012 systematic review of $\mathrm{CBL}$ in health professions education concluded that "overwhelmingly, students enjoy CBL and think it enhances learning" although the empirical data were inconclusive with regard to its effects on learning relative to other approaches. ${ }^{16}$ Our study did demonstrate measurable performance and confidence improvements, although the valid measurement of complex skills and thought pro- cesses is a laudable goal. In discussing problems inherent to the measurement of learning outcomes in medical education, Albanese suggested that even if knowledge acquisition were not improved due to a novel educational modality, the enhanced "work environment for students and faculty is a worthwhile goal in and of itself." ${ }^{\prime 17}$

It is unclear whether the educational benefits attributable to CBL found in this and other studies were due to the means of information delivery (a case), the group learning effect, or both. Nevertheless, educators reportedly enjoy CBL due to its student-engaging and motivational character. ${ }^{16}$ The small group format is certainly demanding in terms of human resources and may be a limiting step for some institutions. Other issues may be related to the methods of implementation (i.e., development of new cases) and faculty preparedness, as CBL is a relatively new and resource-demanding modality 
Table 1. Students' removable partial denture treatment planning comprehension and perceived efficacy during study period and gain associated with each method

\begin{tabular}{|c|c|c|c|c|c|c|}
\hline \multirow[b]{2}{*}{ Assessment } & \multicolumn{4}{|c|}{ Time Point } & \multicolumn{2}{|c|}{$\begin{array}{l}\text { Attributable Gains (Baseline to } \\
2 \text { Weeks) and ANOVA Results }\end{array}$} \\
\hline & $\begin{array}{l}\text { Baseline } \\
\text { Mean (sd) }\end{array}$ & $\begin{array}{l}1 \text { Week } \\
\text { Mean }(\mathrm{sd})\end{array}$ & $\begin{array}{l}2 \text { Weeks } \\
\text { Mean (sd) }\end{array}$ & $\begin{array}{l}6 \text { Months } \\
\text { Mean (sd) }\end{array}$ & Difference & Effect, p-value \\
\hline Comprehension & & & & & $\mathrm{CBL}=31.7$ & Sequence, 0.03 \\
\hline Group $\mathrm{A}(\mathrm{CBL}, \mathrm{LB})$ & $59.3(8.2)$ & $92.4(3.8)$ & $89.6(3.5)$ & $80.5(7.0)$ & $\mathrm{LB}=26.2$ & Method, 0.04 \\
\hline Group B (LB, CBL) & $62.7(10.5)$ & $91.7(4.4)$ & $90.3(4.3)$ & $81.1(8.0)$ & & $\begin{array}{l}\text { Carryover, } 0.05 \\
\text { Period, }<0.0005\end{array}$ \\
\hline Perceived efficacy & & & & & $\mathrm{CBL}=4.3$ & Sequence, 0.5 \\
\hline Group A (CBL, LB) & $2.5(1.6)$ & $6.7(1.4)$ & $7.2(1.2)$ & $5.9(2.0)$ & $\mathrm{LB}=3.6$ & Method, 0.01 \\
\hline Group B (LB, CBL) & $2.8(2.0)$ & $5.9(1.8)$ & $5.9(1.7)$ & $5.4(2.0)$ & & $\begin{array}{c}\text { Carryover, } 0.0006 \\
\text { Period, }<0.0005\end{array}$ \\
\hline
\end{tabular}

$\mathrm{CBL}=$ case-based learning, $\mathrm{LB}=$ lecture-based instruction

Note: Comprehension was determined by objective assessment; perceived efficacy was determined by subjective assessment. Analysis of variance was implemented with Stata's pkcross routine, accounting for the $2 \times 2$ crossover study design features. Sequence refers to intersubject effect due to alternating sequence of instructional method administration. Method refers to intrasubject effect attributable to differences in instructional method. Carryover refers to intrasubject effect due to instructional method that "carries over" from one experimental condition and time point to the next one. Period refers to intrasubject effect attributable to the study period design feature, wherein students in groups A and B differed systematically due to familiarization with experimental (or course and assessment) conditions.

in dental education. A recent study at the University of Florida found that faculty members engaged in a CBL development training course were initially hesitant and uncertain about the method; however, their concerns did not persist after CBL implementation when they "began to consider how to optimize" the method's effectiveness. ${ }^{18}$

The fact that objectively measured comprehension diminished in our study during the followup period, after the end of the course, is a point worth further consideration. Long-term retention of information delivered in didactic courses is an issue relevant to the entirety of health professions education. To some degree, it can be expected that dental students forget information that is not put into practice right away or is perceived to be irrelevant, similar to practicing clinicians whose comprehension of basic sciences knowledge or complex procedures not routinely practiced is likely diminished compared to their own dental school years. ${ }^{19}$ In spite of this phenomenon, our study found that CBL was vastly preferred by the learners (indicating subjective benefits) and had measurable short-term educational benefits (objectively measured within the context of the class). Information/comprehension losses after the course had ended can likely be addressed with higher level, appropriate clinical application and educational experiences or didactic/seminar reinforcements; however, the fact that comprehension decreased over time (as is universally the case, for any learning outcome) does not, in our opinion, detract from the benefits of the approach. It rather reinforces the notion that meaningful information retention must be considered in the context of the revitalization of the entire dental curriculum.

This study's findings should be regarded in acknowledgment of its limitations. These data were generated from one 80-dental student class in one U.S. dental school and may not be generalizable to other schools with different profiles. Furthermore, some variation in CBL implementation (i.e., smallgroup or "instructor" effects) would be expected; however, this does reflect a real-world scenario and, if anything, may have resulted in an attenuation of the CBL benefits that we observed. Further, possible excitement about implementation of a new educational modality may have conferred both objective and subjective knowledge gains - an unavoidable consequence since the intervention could not possibly be masked. It is unknown if and how this study's results might have been different if the six-month follow-up response rate had been higher or lower; the direction of the nonresponse effect is unobservable, while respondents and nonrespondents are groups that are likely systematically different. Finally, we did not use validated instruments to assess RPD treatment 
planning comprehension, efficacy, or method preference; however, these assessments were developed and used by the course director in the context of a predoctoral course and were found to be responsive to change over time and able to discriminate between the educational modalities.

\section{Conclusion}

The results of this study at one U.S. dental school provide additional support for students' overwhelming preference of CBL over traditional, lecture-based instruction. The increase in students' RPD treatment planning confidence found in this study represents a meaningful endpoint of didactic instruction and may correspond to improved performance in student clinics. As data on the benefits of CBL in dental education accumulate, we recommend that its use be considered for preclinical dental courses along with other active learning modalities as means to optimize the dental education environment, including taking account of students' learning outcomes and preferences.

\section{Acknowledgments}

This study was supported by funding from the 2014-15 ADEA Gies Foundation/AADR Academic Dental Careers Fellowship Program.

\section{Disclosure}

The authors declared no conflicts of interest.

\section{REFERENCES}

1. Hendricson WD, Andrieu SC, Chadwick DG, et al. Educational strategies associated with development of problem-solving, critical thinking, and self-directed learning. J Dent Educ 2006;70(9):925-36.

2. Pyle M, Andrieu SC, Chadwick DG, et al. The case for change in dental education. J Dent Educ 2006;70(9):921-4.

3. Divaris K, Barlow PJ, Chendea SA, et al. The academic environment: the students' perspective. Eur J Dent Educ 2008;12(Suppl 1):120-30.

4. Murphy RJ, Gray SA, Straja SR, Bogert MC. Student learning preferences and teaching implications. J Dent Educ 2004;68(8):859-66.
5. Victoroff KZ, Hogan S. Students' perceptions of effective learning experiences in dental school: a qualitative study using a critical incident technique. J Dent Educ 2006;70(2):124-32.

6. Michael J. Where's the evidence that active learning works? Adv Physiol Educ 2006;30:159-67.

7. Nadershahi NA, Bender DJ, Beck L, et al. An overview of case-based and problem-based learning methodologies for dental education. J Dent Educ 2013;77(10):1300-5.

8. Keeve PL, Gerhards U, Arnold WA, et al. Job requirements compared to dental school education: impact of a case-based learning curriculum. GMS Z Med Ausbild 2012;29:Doc54.

9. McKenzie CT. Dental student perceptions of case-based educational effectiveness. J Dent Educ 2013;77(6):688-94.

10. Zhang SY. Case-based learning in clinical courses in a Chinese college of stomatology. J Dent Educ 2012;76(10):1389-92.

11. Kumar V, Gadbury-Amyot CC. A case-based and teambased learning model in oral and maxillofacial radiology. J Dent Educ 2012;76(3):330-7.

12. Echeto LF, Sposetti V, Childs G, et al. Evaluation of team-based learning and traditional instruction in teaching removable partial denture concepts. J Dent Educ 2015;79(9):1040-8.

13. Srinivasan M, Wilkes M, Stevenson F, et al. Comparing problem-based learning with case-based learning: effects of a major curricular shift at two institutions. Acad Med 2007;82:74-82.

14. Wellek S, Blettner M. On the proper use of the crossover design in clinical trials: part 18 of a series on evaluation of scientific publications. Dtsch Arztebl Int 2012;109: 276-81.

15. Faul F, Erdfelder E, Buchner A, Lang AG. Statistical power analyses using $\mathrm{G}^{*}$ Power 3.1: tests for correlation and regression analyses. Behav Res Methods 2009;1: 149-60.

16. Thistlethwaite JE, Davies D, Ekeocha S, et al. The effectiveness of case-based learning in health professional education: a BEME systematic review. BEME guide no. 23. Med Teach 2012;34:e421-44.

17. Albanese M. Problem-based learning: why curricula are likely to show little effect on knowledge and clinical skills. Med Educ 2000;34:729-38.

18. Behar-Horenstein LS, Catalanotto FA, Nascimento MM. Anticipated and actual implementation of case-based learning by dental faculty members during and after training. J Dent Educ 2015;79(9):1049-60.

19. Custers EJ. Long-term retention of basic science knowledge: a review study. Adv Health Sci Educ Theory Pract 2010;15(1):109-28. 